Psicologia Escolar

e Educacional
ARTIGO

DOI: http://dx.doi.org/10.1590/2175-35392020215946

Elocid - e215946

\title{
MARCOS DO COMPORTAMENTO VERBAL E INTERVENÇÃO COMPORTAMENTAL INTENSIVA EM TRIGÊMEOS COM AUTISMO
}

\author{
Suelen Priscila Macedo Farias ${ }^{1} \mathbb{D}$; Nassim Chamel Elias ${ }^{1} \mathbb{D}$
}

\begin{abstract}
RESUMO
O Transtorno do Espectro do Autismo é caracterizado por dificuldades em comunicação e interação social e interesses e comportamentos restritos, repetitivos e estereotipados. A intervenção comportamental intensiva tem trazido resultados promissores na intervenção com esse público. 0 objetivo foi verificar os efeitos do ensino de múltiplos operantes verbais no desenvolvimento de repertórios em trigêmeos dentro do espectro com 3 anos e 6 meses de idade no início do estudo. A intervenção durou 12 meses, num total de oito horas semanais, e foi planejada de acordo com dados da primeira avaliação. Foi utilizado o delineamento de pré e pós testes. Os resultados indicaram que a intervenção foi efetiva, sendo que o participante com menor comprometimento adquiriu mais repertórios. A diferença dos resultados indica que os ganhos obtidos não foram em função da passagem do tempo, mas indica uma relação com os repertórios iniciais e com o número de programas aplicados.
\end{abstract}

Palavras-chave: Análise do Comportamento; Linguagem; Autismo.

\section{Verbal Behavior Milestones and Intensive Behavioral Intervention in Triplets with Autism}

\begin{abstract}
The Autism Spectrum Disorder is characterized by difficulties in communication and social interaction and restricted, repetitive and stereotyped interests and behaviors. Intensive behavioral intervention has brought promising results in the intervention with this audience. The objective was to verify the effects of teaching multiple verbal operant on the development of repertoires in triplets within the spectrum at the age of 3 years and 6 months at the beginning of the study. The intervention lasted 12 months, for a total of eight hours a week, and was planned according to data from the first assessment. Pre- and post-test design was used. The results indicated that the intervention was effective, and the participant with the lower commitment acquired more repertoires. The difference in the results indicates that the gains obtained were not due to the passage of time, but indicates a relationship with the initial repertoires and the number of programs applied.
\end{abstract}

Keywords: Behavior Analysis; Language; Autism.

\section{Marcos del comportamiento verbal e intervención comportamental intensiva en trillizos con autismo}

\section{RESUMEN}

El Trastorno del Espectro del Autismo es caracterizado por dificultades en comunicación e interacción social e intereses y comportamientos restrictos, repetitivos y estereotipados. La intervención comportamental intensiva ha traído resultados promisores en la intervención con ese público. El objetivo fue verificar los efectos de la enseñanza de múltiples operantes verbales en el desarrollo de repertorios en trillizos dentro del espectro con 3 años y 6 meses de edad en el inicio del estudio. La intervención tuvo duración de 12 meses, en un total de ocho horas semanales, y fue planificada de acuerdo con datos de la primera evaluación. Se utilizó el delineamiento de antes y tras testes. Los resultados apuntaron que la intervención fue efectiva, siendo que el participante con menor comprometimiento adquirió más repertorios. La diferencia de los resultados indica que las ganancias obtenidos no fueron función del paso del tiempo, pero indica una relación con los repertorios iniciales y con el número de programas aplicados.

Palabras clave: Análisis de la Conducta; Lenguaje; Autismo.

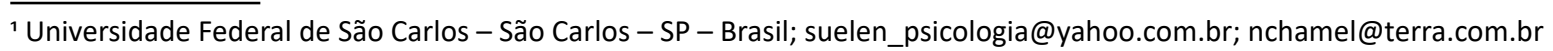




\section{INTRODUÇÃO}

A prevalência de casos de Transtorno do Espectro do Autismo (TEA) tem crescido de forma significativa em todo o mundo, especialmente durante as últimas décadas. De acordo com o Centers for Disease Control and Prevention (CDC) dos Estados Unidos da América, em 2014, a prevalência encontrada de TEA foi de uma em cada 59 crianças com oito anos de idade. O TEA é caracterizado por alterações comportamentais em múltiplos contextos que se manifestam desde o início do crescimento e que ocasionam prejuízos variados na interação social e na comunicação com padrões restritos e repetitivos de comportamento e interesse (APA, 2013).

Lovaas (1987) e Werner, Dawson, Munson e Osterling (2005) destacam que a identificação do transtorno na primeira infância propicia a estimulação precoce e um prognóstico diferencial para essas crianças. Esses autores demonstraram que a efetividade das intervenções comportamentais, quando bem delineadas, sistematizadas e intensivas, permitem uma aquisição mais rápida dos comportamentos-alvo e, dessa forma, promovem a diminuição nos custos do tratamento para as famílias e para o governo. Nesse sentido, para esta pesquisa, optou-se pela intervenção comportamental, por se tratar de uma ciência com comprovação científica de sua eficácia (Howard, Stanislaw, Green, Sparkman, \& Cohen, 2014; Lovaas, 1987).

Lovaas (1987) conduziu o estudo pioneiro sobre intervenção comportamental intensiva aplicada a indivíduos com diagnóstico de autismo. Os participantes foram crianças com autismo com menos de 4 anos de idade no início do estudo, divididas em três grupos, um grupo experimental e dois grupos controles. $O$ grupo experimental, exposto à intervenção comportamental intensiva, foi composto por 19 crianças. Cada criança era atendida por um educador por aproximadamente 40 horas semanais. Um grupo controle, composto por 19 crianças, recebeu intervenção comportamental por, no máximo, 10 horas semanais. 0 outro grupo controle era composto por 21 crianças matriculadas em centros de atendimento que não utilizavam intervenção comportamental nem intervenção intensiva. Foram utilizadas diferentes escalas para medir os repertórios dos participantes antes e após o período de intervenção, que durou aproximadamente dois anos. Os resultados indicaram que $47 \%$ das crianças do grupo experimental apresentaram desenvolvimento próximo ao esperado para uma criança com desenvolvimento típico de mesma faixa etária. Por outro lado, apenas $2 \%$ das crianças dos grupos controles apresentaram desenvolvimento próximo ao esperado para a idade. Os dados de Lovaas (1987) sugerem que a intervenção comportamental intensiva, aplicada por pelo menos dois anos consecutivos, pode produzir ganhos comportamentais significativos para crianças com TEA.
No Brasil, o primeiro e único estudo até o momento nessa área foi publicado por Gomes, de Souza, Silveira, \& Oliveira (2017). O objetivo desse estudo foi avaliar os efeitos de um ano de intervenção comportamental intensiva na aquisição de novos comportamentos por nove crianças brasileiras com autismo, com idades entre 1 ano e 3 meses e 2 anos e 11 meses. Foram realizadas avaliações individuais, utilizando o Perfil Psicoeducacional Revisado (PEP-R) (Schopler, Reichler, Bashford, Lansing, \& Marcus, 1990) e o Inventário Portage Operacionalizado (IPO) (Williams \& Aiello, 2001), antes e após a introdução da intervenção. Esses instrumentos medem o desenvolvimento das crianças em diversas áreas e permitem a realização de comparações com o desempenho esperado para a idade cronológica. A intervenção foi conduzida por aproximadamente 15 horas semanais na residência dos participantes. Os resultados, analisados individualmente, indicaram que os participantes apresentaram novas habilidades em todas as áreas avaliadas, mesmo que em graus variados. Segundo Gomes et al. (2017), considerando os resultados obtidos no PEP-R, os

desempenhos mais baixos foram observados nas áreas de desempenho cognitivo e cognitivo verbal, provavelmente porque essas áreas englobam habilidades... diretamente relacionadas ao repertório de fala e que precisariam de mais tempo para serem refinadas, além do primeiro ano de intervenção. (pp. 383-384).

Os resultados obtidos por Lovaas (1987) e Gomes et al. (2017) são indicativos da efetividade da intervenção comportamental intensiva aplicada a crianças com TEA. Entretanto, duas questões ainda permeiam essa área de pesquisa: a intensidade da intervenção e as características dos participantes. Lovaas sugere 40 horas de intervenção semanal por pelo menos 24 meses, enquanto Gomes e colaboradores obtiveram resultados significativos com 15 horas semanais de intervenção por aproximadamente 12 meses. Nesse sentido, um aspecto que deve ser levado em consideração para que os resultados se efetivem refere-se aos repertórios iniciais dos participantes, como presença de comportamento verbal vocal (fala) e comportamentos inadequados, entre outros. Nos dois estudos citados, por contarem com a participação de várias crianças, as características e a história de vida individuais são pouco exploradas.

Considerando a efetividade das intervenções comportamentais intensivas em crianças com TEA e os déficits em comunicação presentes nessa população, esse estudo teve o objetivo de verificar os efeitos do ensino de múltiplos operantes verbais simultaneamente no desenvolvimento de novos repertórios em trigêmeos com TEA com 3 anos e 6 meses de idade no início do estudo. A intervenção durou 12 meses e era conduzida 
todos os dias da semana, num total de oito horas semanais.

\section{MÉTODO}

\section{Delineamento Experimental}

Foi utilizado um delineamento de pré e pós teste com a aplicação do VB-MAPP (Verbal Behavior Milestones Assessment and Placement Program; Sundberg, 2008). O procedimento foi aplicado de forma individualizada com cada participante.

\section{Participantes}

Os participantes foram trigêmeos com diagnóstico de TEA. Os trigêmeos foram gerados em duas placentas diferentes, sendo que em uma placenta havia dois fetos de gêmeos univitelínicos (T1 e T2), que nasceram primeiro, apresentam maiores comprometimentos e foram diagnosticados com TEA aos 2 anos e 9 meses por uma neuropediatra. Na outra placenta havia apenas um feto (T3), que nasceu por último e foi diagnosticado com TEA aos 2 anos e 11 meses pela mesma neuropediatra; ele apresenta menores comprometimentos. Os trigêmeos foram gerados a partir de uma fertilização in vitro e nasceram com 26 semanas de gestação via parto cesárea. No início da pesquisa, eles tinham 3 anos e 5 meses de idade, ao final, 5 anos e 2 meses.

T1 e T2 tiveram diagnóstico pelo CID F84.0, quadro de autismo clássico e atraso global do desenvolvimento neuropsicomotor. Eles andaram aos 2 anos e 4 meses. Em relação à coordenação motora, eles chutavam bola sem direção adequada, subiam em cadeiras, mas não subiam escada sem apoio, corriam com dificuldade, não levavam colher a boca, faziam rabiscos, mas não esboçavam figura humana, não empilhavam objetos e não folheavam páginas de livros. Quanto às habilidades cognitivas, eles não relacionavam objetos com o seu uso, não brincavam de esconde-esconde. Em relação às habilidades sócio afetivas, eles não reconheciam pessoas da família. Não apresentavam fala e não possuíam controle de esfíncteres.

T3 teve diagnóstico pelo CID F84.0, quadro de Transtorno do Espectro do Autismo. Ele fazia pouco contato visual, não atendia ordens e apresentava atraso na comunicação verbal e não verbal, apresentava ecolalia, disfunção sensorial auditiva e restrição alimentar, pouco uso da imaginação e do simbolismo. Quanto ao desenvolvimento neuropsicomotor, corria, pulava, subia e descia escada, mas com dificuldade. Quanto à coordenação motora fina, fazia encaixes de formas geométricas, fazia rabiscos, apresentava dificuldade em manusear talheres, transferia líquidos e objetos de um recipiente para o outro. Quanto à linguagem, falava palavras soltas, não fazia reconto de histórias, não conhecia gêneros masculino e feminino. Quanto à cognição, conhecia cores, estava aprendendo noção de tamanho e de letras, montava quebra-cabeça, cantava uma música e fazia associações adequadamente. Todas essas informações são de acordo com o laudo da neuropediatra no início da pesquisa.

\section{Instrumento}

Para as medidas de pré e pós intervenção, foi utilizado o VB-MAPP. Segundo Sundberg (2008), para desenvolver o VB-MAPP, foi considerada a análise skinneriana (Skinner, 1957) de comportamento verbal para a criação de um protocolo de avaliação de comportamentos sociais e de linguagem para crianças com TEA ou atrasos similares e permite determinar quais desses comportamentos estão presentes ou em atraso. Essas informações são, então, utilizadas para elencar os comportamentos que farão parte do programa de ensino para aquela criança.

O VB-MAPP é dividido em três níveis, de acordo com determinados marcos de desenvolvimento (Nível 1: 0-18 meses; Nível 2: 18-30 meses; Nível 3: 30-48 meses). 0 Nível 1 avalia o repertório de mandos, tatos, comportamentos de ouvinte, habilidades visuais e percepção visual, brincar independentemente, habilidades sociais, imitação, ecoico e vocalização espontânea. 0 Nível 2 continua avaliando as habilidades listadas acima, além de avaliar o comportamento de ouvinte em relação à característica, função e classe dos estímulos (LRFCC), habilidades intraverbais e desempenho em situações de grupo e rotina. O Nível 3 avalia todas as habilidades descritas acima além de leitura, escrita e matemática. Esses repertórios podem ser medidos por observação da criança em ambiente natural ou em situações controladas e, a depender do desempenho, a criança recebe " 0 ", " 0,5 " ou " 1 " ponto para o item sendo avaliado. Ao ultrapassar 5 pontos em um determinado operante, a criança vai para o nível seguinte, ou seja, a criança pode estar, por exemplo, no Nível 1 para mando e no Nível 2 para tato.

\section{Procedimento}

Após aprovação pelo Comitê de Ética em Pesquisa em Seres Humanos (Parecer número 2.016.125) da Universidade Federal de São Carlos, os pais dos trigêmeos foram contatados para convidar os trigêmeos a participarem da pesquisa. Após a concordância dos mesmos e assinatura do Termo de Consentimento Livre e Esclarecido (TLCE), foi aplicada a primeira avaliação utilizando o VB-MAPP. Não foi apresentado o Termo de Assentimento para as crianças, pois entende-se que elas não apresentavam os pré-requisitos mínimos para leitura, entendimento do conteúdo (mesmo que apresentado de forma oral) e do significado de um Termo de Assentimento.

Os programas de intervenção comportamental foram planejados e modificados de forma individualizada de acordo com os pontos fortes e déficits específicos de 
cada participante de acordo com cada avaliação pelo VB-MAPP. A primeira aplicação foi realizada antes do início da intervenção. Decorridos oito meses de intervenção, os trigêmeos foram reavaliados pelo VB-MAPP para verificar as possíveis evoluções e acompanhar o desenvolvimento dos participantes. Decorridos mais quatro meses de intervenção (12 meses após a aplicação da primeira avaliação), foi realizada uma terceira avaliação com o VB-MAPP.

\section{Intervenção Comportamental Intensiva}

Os programas foram aplicados por duas estudantes de psicologia (Estudante A e Estudante B). A Estudante A estava no 40 período do curso e a Estudante B no 8ㅇ período, no início da pesquisa. As estudantes receberam treinamento em Análise Aplicada do Comportamento pela primeira autora para a realização dos procedimentos com o objetivo de padronizar e uniformizar a prática com os participantes. $O$ treinamento teve duração de 40 horas, sendo 10 horas de teoria e 30 horas de prática. As atividades práticas foram divididas da seguinte forma, na residência dos participantes: 10 horas de observação da primeira autora aplicando os programas com os participantes; 10 horas de observação e execução, em que havia intercalação entre observar a primeira autora aplicando os programas e aplicar os programas com supervisão da primeira autora; e 10 horas finais de execução em que as estudantes aplicavam os programas com supervisão da primeira autora.

Todas as aplicações do VB-MAPP foram feitas pela primeira autora. A Estudante $A$ realizou a intervenção com T1 e a Estudante 2 com T2 e T3. A intervenção era feita todos os dias da semana, de segunda à sexta, sendo 1 hora e 30 minutos para cada criança por dia, totalizando oito horas semanais. A primeira autora acompanhava presencialmente a aplicação dos programas uma vez por semana na casa dos participantes e realizava supervisões de duas horas semanais com as estudantes para discutir o desenvolvimento dos programas, a evolução dos participantes, a mudança de protocolos e a adoção de novas estratégias de ensino (quando necessárias).

Após a primeira aplicação do VB-MAPP, foi feita uma avaliação de preferência com múltiplos estímulos (DeLeon \& Iwata, 1996) para identificar os itens de maior preferência de cada participante para serem usados como consequências em tentativas de ensino. A avaliação de preferência foi repetida ao início de cada semana.

$O$ ensino se deu por meio do uso de reforçamento diferencial e da introdução e esvanecimento de dicas e ajudas que visam minimizar a ocorrência de erros e aumentar o acesso aos itens de preferência. Para os programas de comportamento de ouvinte, a hierarquia de dicas foi a seguinte: ajuda física total, ajuda física leve, dica gestual e sem dica ou ajuda. Para os programas de linguagem, foram utilizadas a dica ecoica imediata, a dica ecoica após dois segundos, a dica ecoica após cinco segundos e sem dica. O esvanecimento das dicas e ajudas estava condicionado ao desempenho de cada participante. $O$ critério de aprendizagem estabelecido como condição para avançar nas fases de ensino (novo programa ou retirada gradual da dica) era o participante ter um percentual de $90 \%$ de respostas corretas independentes em três blocos consecutivos da mesma habilidade.

Cada programa foi apresentado em blocos de nove tentativas, todos executados no mesmo dia, sendo, inicialmente, 10 minutos de intervenção e 5 minutos de intervalo. Gradualmente, esse tempo foi aumentado para 20 minutos de intervenção e 5 de intervalo para T1 e T2 e 30 minutos de intervenção e 5 de intervalo para T3. Os intervalos eram conduzidos com base no repertório de brincar identificado pelo VB-MAPP. Os participantes eram estimulados a brincar de forma funcional e livre durante o intervalo.

As respostas corretas em cada programa produziam acesso ao item de preferência e reforço social ("ótimo, você é muito bom, demais, parabéns", seguido de cócegas) fornecido pelas estudantes. Para garantir a operação estabelecedora de privação e assim aumentar as chances de os itens de preferência manterem o valor reforçador, nos dias das sessões de ensino, a família era instruída a manter esses itens fora do alcance das crianças.

A Tabela 1 apresenta as habilidades indicadas no VB-MAPP e os programas aplicados a cada participante. Para T1 e T2, para o repertório de mando, foi selecionada a resposta de apontar, pois eles não apresentavam respostas vocais.

Para o ensino das respostas contidas nos programas, foi utilizado o Ensino por Tentativas Discretas (DTT, do inglês Discrete Trial Teaching). O DTT consiste em (i) obter a atenção do aprendiz, (ii) apresentar uma instrução objetiva e os materiais (a depender do programa), (iii) aguardar a resposta do aprendiz, (iv) apresentar consequências diferenciais para respostas corretas e incorretas e (v) registrar a resposta. Um intervalo em segundos entre as tentativas determina o término de uma tentativa e o início da próxima (Smith, 2001).

\section{RESULTADOS}

Os resultados da primeira aplicação do VB-MAPP indicaram que os trigêmeos se encontravam no Nível 1. T1 e T2 continuaram no Nível 1 do VB-MAPP até a terceira aplicação, mas apresentaram melhora em todos os repertórios de seus programas. T3 alcançou o Nível 3 do VB-MAPP na terceira avaliação. Os Quadros 1, 2 e 3 apresentam, respectivamente, os resultados alcançados por T1, T2 e T3 nas três aplicações do VB-MAPP. As células em cinza claro dos quadros correspondem aos resultados na primeira aplicação; as células em cinza escuro na segunda aplicação; e as quadriculadas na terceira aplicação. De maneira geral, nota-se ganhos em 
Tabela 1. Habilidades ensinadas para cada participante.

\begin{tabular}{|c|c|c|}
\hline Habilidade do VB-MAPP & Participante & Programa \\
\hline \multirow{2}{*}{ 1. Mando } & T1 e T2 & Apontar estímulo \\
\hline & T3 & Fazer pedido com frases \\
\hline \multirow{2}{*}{ 2. Tato } & T1 e T2 & Nomeação de pessoas familiares \\
\hline & T3 & Nomeação de objetos / ações \\
\hline \multirow{2}{*}{ 3. Comportamento de Ouvinte } & T1 e T2 & $\begin{array}{l}\text { Contato Visual / Sentar adequado / Identificação de pessoas } \\
\text { familiares }\end{array}$ \\
\hline & T3 & $\begin{array}{l}\text { Contato Visual / Sentar adequado / Identificação de objetos } \\
\text { e da função de objetos }\end{array}$ \\
\hline \multirow{2}{*}{ Habilidades Visuais e MTS } & T1 e T2 & Olhar para objeto / Pareamento simples \\
\hline & T3 & Olhar para objeto / Pareamento complexo \\
\hline 5. Brincar independente & T1, T2 e T3 & Imitação Motora com Objeto \\
\hline \multirow{2}{*}{ Comportamento e Brincar social } & T1 e T2 & Seguir Instrução Simples \\
\hline & T3 & Seguir Instrução de três ordens \\
\hline \multirow{3}{*}{ 7. Imitação Motora } & T1 e T2 & Imitação Motora Grossa / Imitação Grafomotora (traços) \\
\hline & \multirow{2}{*}{ T3 } & Imitação Motora de três passos, Motora Oral e Motora \\
\hline & & Fina / Imitação Grafomotora (letras) \\
\hline 8. Vocalização Espontânea & T1, T2 e T3 & Ecoico \\
\hline 9. Função, Classe e Característica & $\mathrm{T} 3$ & Identificação e Nomeação por Função, Classe e Característica \\
\hline 10. Intraverbal & T3 & Conversação \\
\hline $\begin{array}{l}\text { 11. Rotina de Classe e Habilidades } \\
\text { em Grupo }\end{array}$ & T3 & Imitação com objetos / Seguir Instrução \\
\hline
\end{tabular}

Fonte: Dados da pesquisa.

todos os repertórios avaliados para os três participantes.

As Figuras 1 e 2 apresentam, respectivamente, 0 desempenho de T1 e T2 e as Figuras 3 e 4 apresentam o desempenho de T3. A partir do registro da aplicação das sessões de DTT, foi criado um gráfico cumulativo no Excel, sendo que cada ponto corresponde ao número de respostas corretas em um bloco de nove tentativas somado ao número de respostas do ponto anterior. Foram utilizadas as respostas registradas ao longo dos 50 primeiros blocos de ensino de cada repertório, por já permitir, por inspeção visual, identificar a curva de aprendizagem em cada repertório. Nota-se, pelos dados apresentados nessas figuras, que $\mathrm{T} 1$ e T2 apresentam curvas de aprendizagem mais acentuadas desde o início da intervenção para os repertórios de seguir instrução, contato visual e imitação com objeto; T3 apresenta curvas de aprendizagem desde o início da intervenção para todos os repertórios.

\section{DISCUSSÃO}

A melhora nos desempenhos dos participantes identificada nas aplicações do VB-MAPP sugere a efe- tividade e a eficácia da intervenção comportamental intensiva. A diferença dos resultados de $\mathrm{T} 3$ em relação a T1 e T2 indica que os ganhos obtidos não foram somente função da passagem do tempo (maturação), mas indica uma relação com os repertórios iniciais e com o número de programas aplicados a cada participante e do próprio desempenho em cada programa. Bloh (2008) demonstrou a importância do repertório de entrada em detrimento à idade no ensino de tatos para cinco participantes com idades entre seis e 21 anos, em que somente aqueles que já possuíam repertórios verbais iniciais alcançaram o critério de aprendizagem.

Os dados da presente pesquisa replicam aqueles encontrados por Lovaas (1987) e Gomes et al. (2017), em que há uma grande variabilidade entre os desempenhos finais de cada participante. Nesse sentido, pode-se inferir que a intensidade da intervenção comportamental depende de vários fatores individuais e ambientais e que não deve ser definida de forma única e universal. Além de corroborar a afirmação de Gomes et al. (2017) de que a presença da fala (como é o caso de T3) pode estar relacionada com a aquisição mais acelerada de 


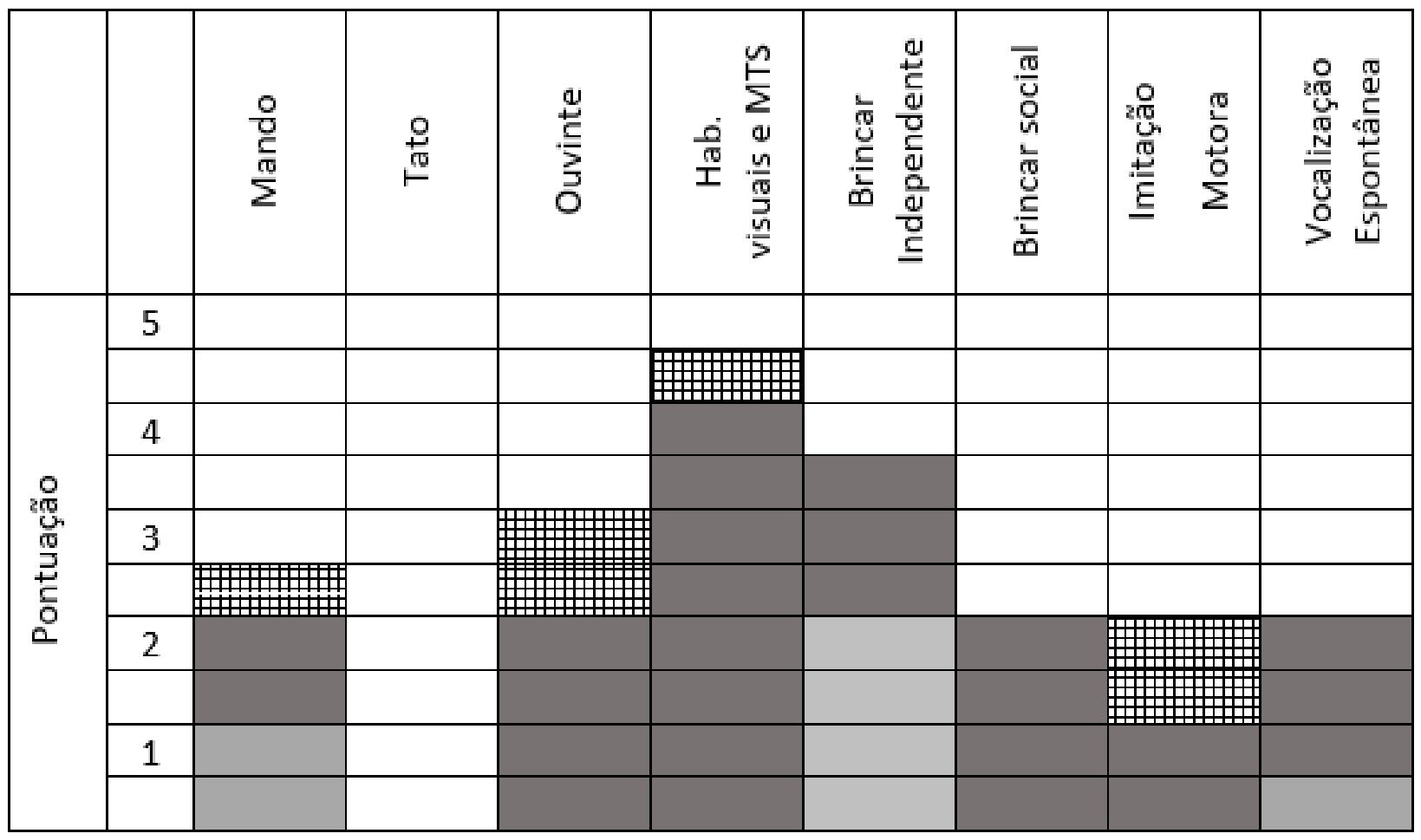

Quadro 1. Resultados obtidos por T1 nas três aplicações do VB-MAPP.

Nota: As células em cinza claro correspondem aos resultados na primeira aplicação; as células em cinza escuro na segunda aplicação; e as quadriculadas na terceira aplicação.

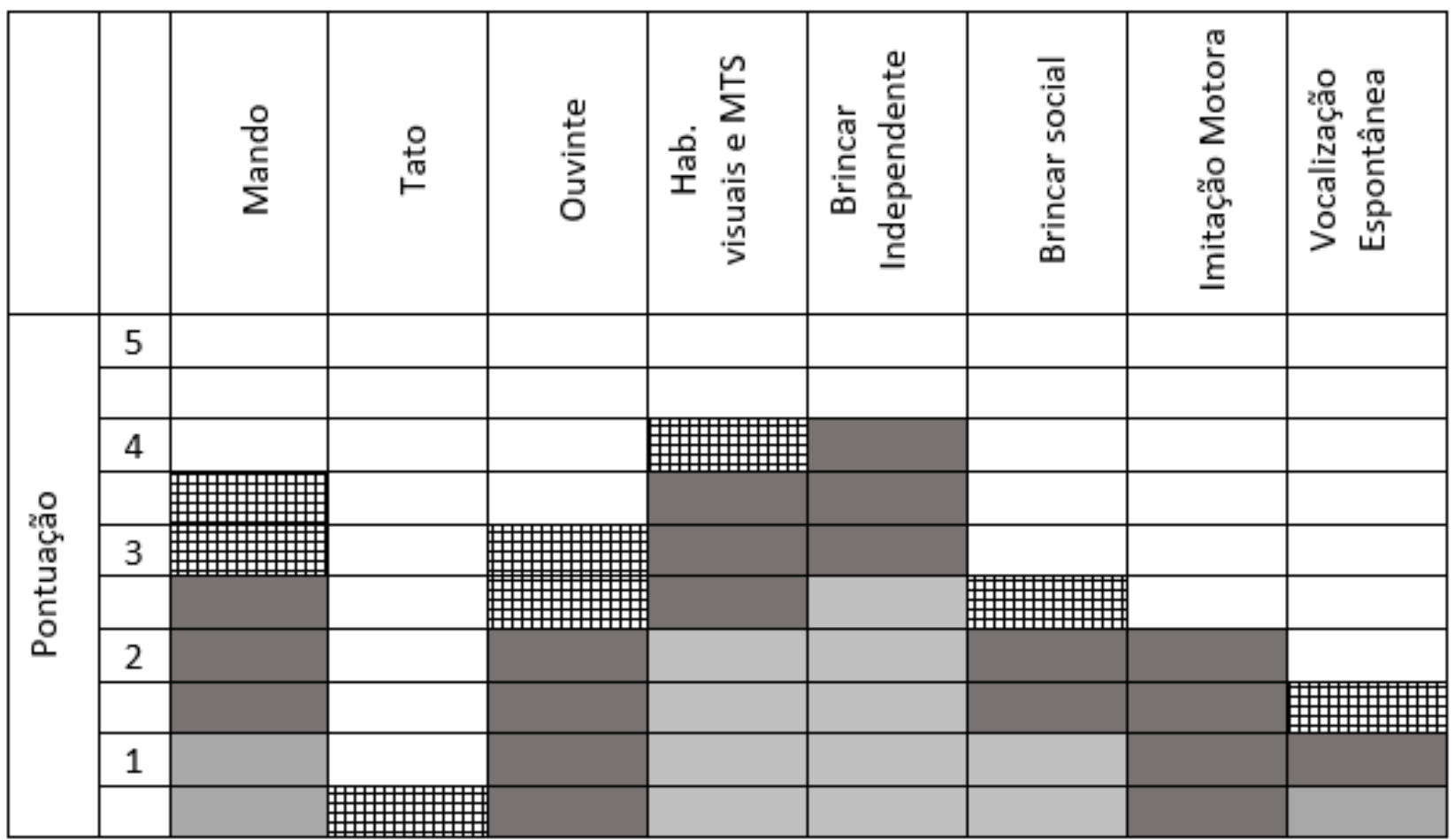

Quadro 2. Resultados obtidos por T2 nas três aplicações do VB-MAPP.

Nota: As células em cinza claro correspondem aos resultados na primeira aplicação; as células em cinza escuro na segunda aplicação; e as quadriculadas na terceira aplicação. 


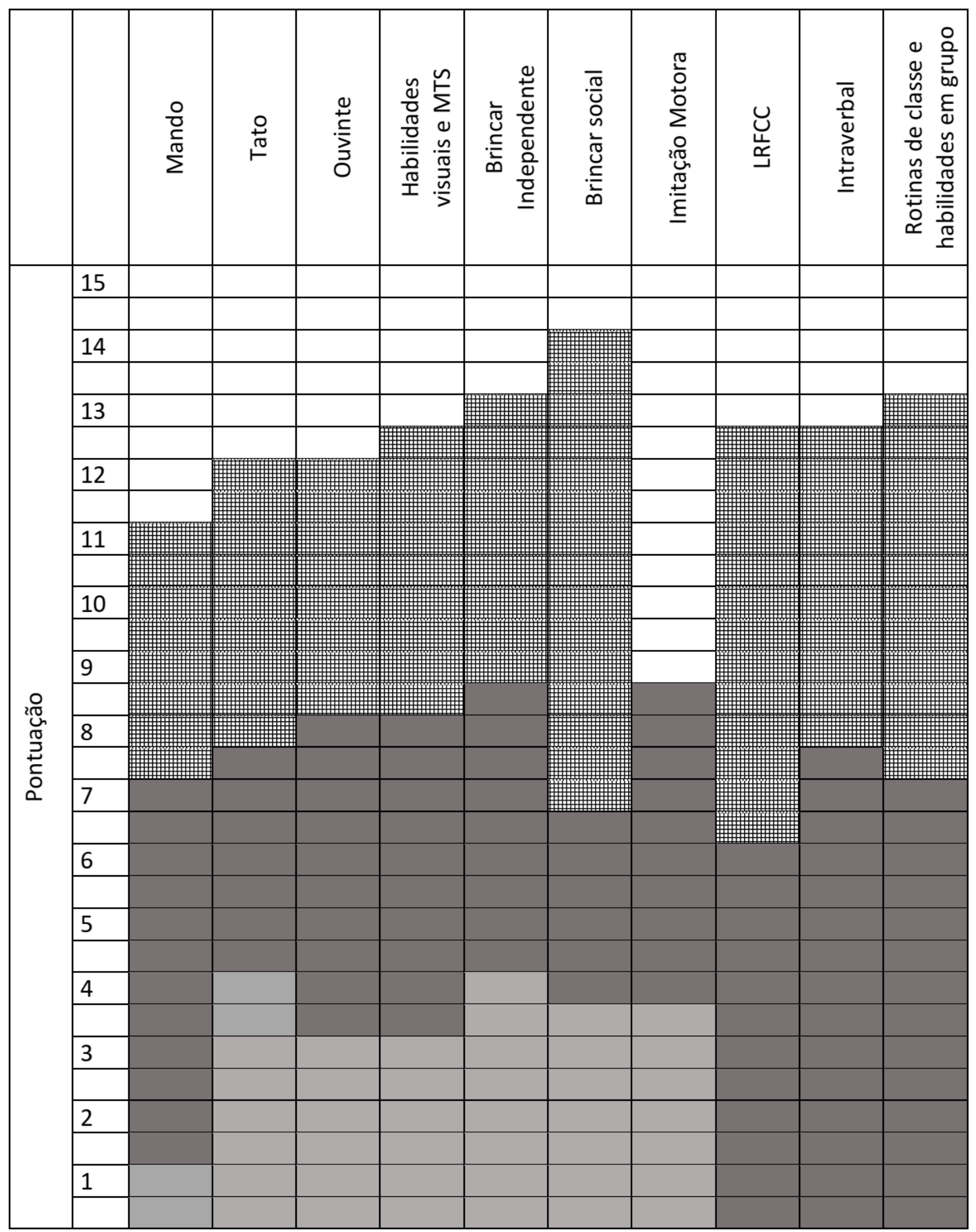

Quadro 3. Resultados obtidos por T3 nas três aplicações do VB-MAPP.

Nota: As células em cinza claro correspondem aos resultados na primeira aplicação; as células em cinza escuro na segunda aplicação; e as quadriculadas na terceira aplicação. 
alguns repertórios, principalmente, os que envolvem comportamento verbal.

Nessa pesquisa, os trigêmeos foram expostos ao mesmo número de horas de intervenção semanal. Entretanto, nota-se, de acordo com os dados das Figuras 1 e 2, que o processo de aprendizagem de T1 e T2, com diagnóstico que indica déficits iniciais mais severos, é mais lento em comparação com T3, cujos déficits iniciais são menos acentuados. Esses padrões idiossincráticos já foram identificados na literatura de aquisição de novas habilidades (Volkert, Lerman, Trosclair, Addison, \& Kodak, 2008).

Essa diferença na aquisição de novas habilidades pode também estar relacionada com a aquisição de re-

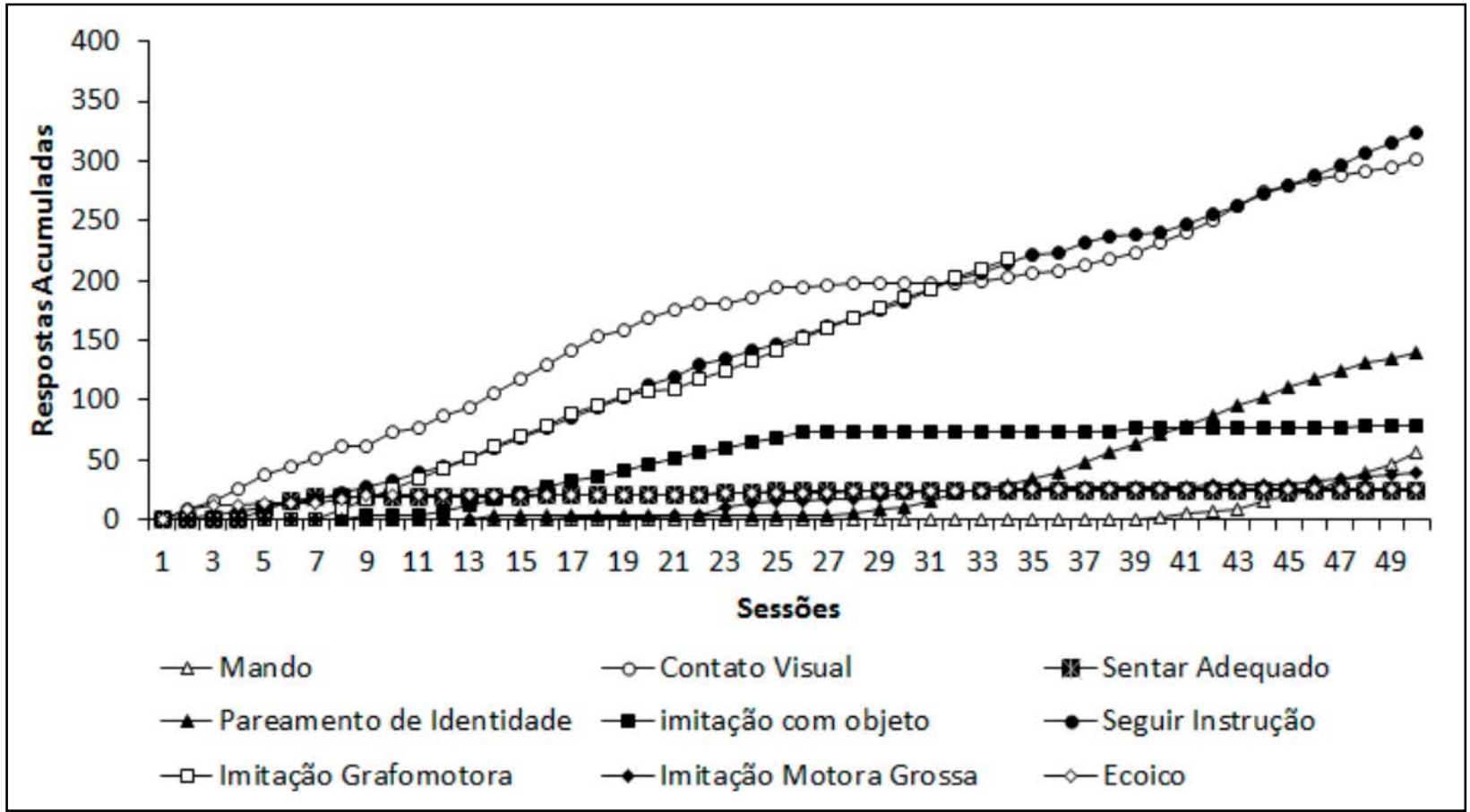

Figura 1. Número de respostas acumuladas independentes de T1 nas primeiras 50 sessões.

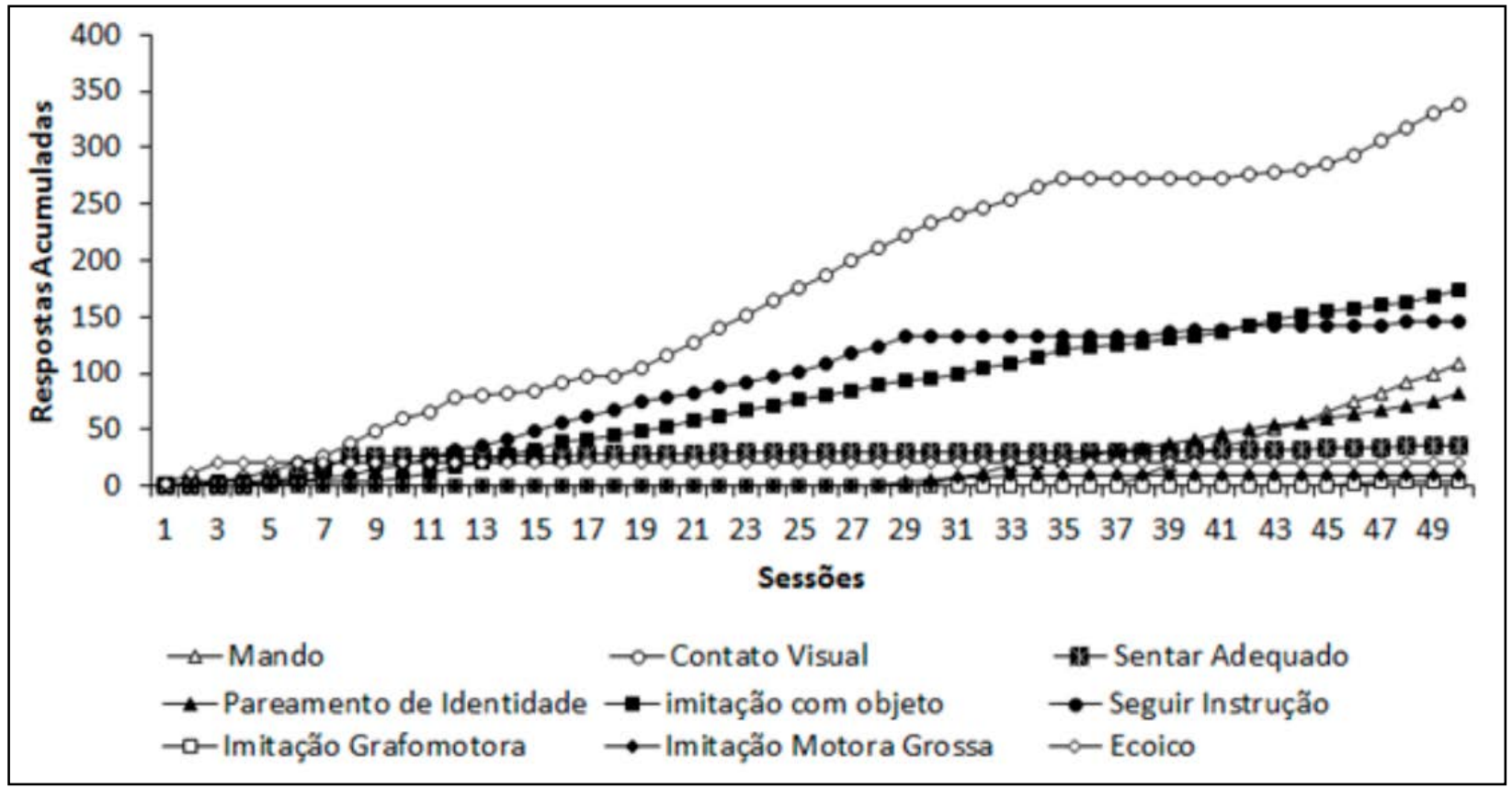

Figura 2. Número de respostas acumuladas independentes de T2 nas primeiras 50 sessões. 
pertório verbal. O participante que obteve os melhores resultados (T3) adquiriu repertórios de ecoico e de mando (ver Figura 3) logo nas primeiras sessões, enquanto os outros dois participantes mostraram evidência da aquisição desses operantes a partir, somente, da sessão 45 (ver Figuras 1 e 2). Alguns estudos têm sugerido que o sucesso nesse domínio parece ser um forte preditor de melhores resultados globais (Szatmari, Bryson,
Boyle, Streiner, \& Duku, 2003; Venter, Lord, \& Schopler, 1992). Martone e Santos-Carvalho (2012) realizaram uma revisão de literatura sobre comportamento verbal e autismo e identificaram que a eficácia do procedimento de ensino foi maior em casos em que os participantes já haviam adquirido comportamento verbal.

Adicionalmente, T3 adquiriu todas as habilidades presentes no Nível 1 e Nível 2 do VB-MAPP, que en-

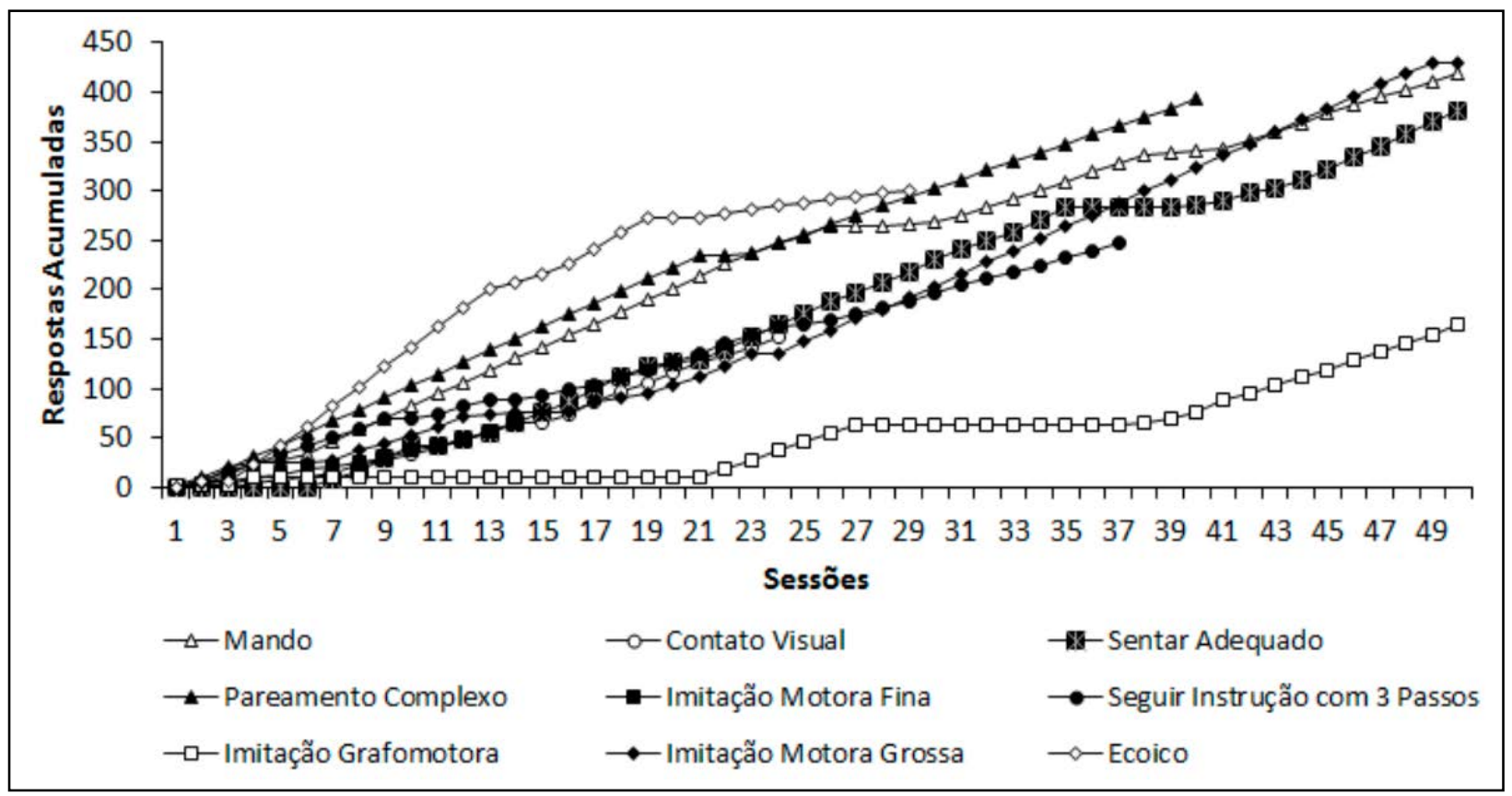

Figura 3. Número de respostas acumuladas independentes de T3 nas primeiras 50 sessões para os repertórios iniciais.

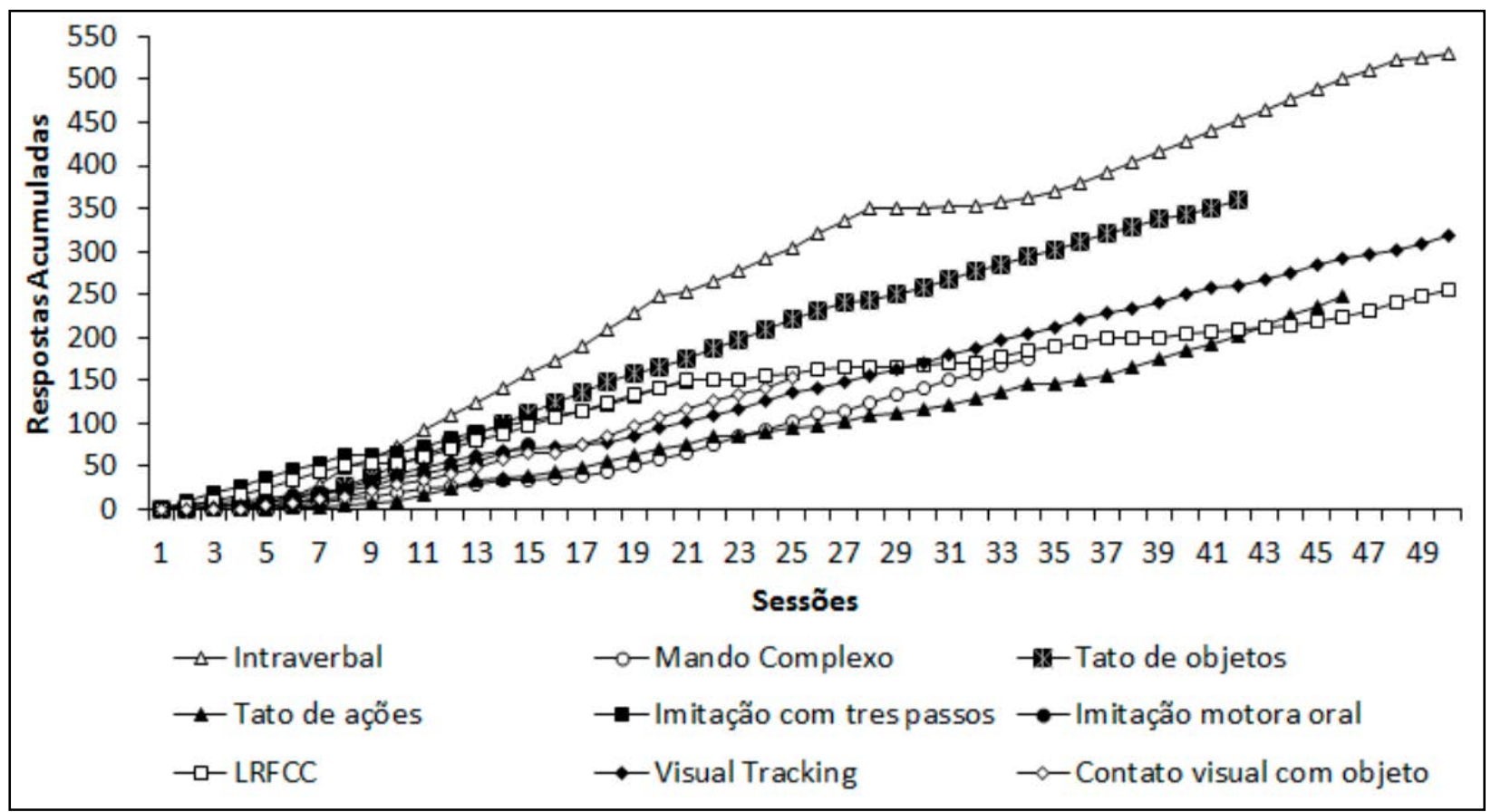

Figura 4. Número de respostas acumuladas independentes de T3 nas primeiras 50 sessões para os repertórios mais complexos. 
volviam tanto repertórios de falante como de ouvinte. Embora a aquisição desses repertórios, no início da aquisição de comportamento verbal, ocorra de forma independente (Skinner, 1957), a integração entre esses repertórios pode facilitar a aprendizagem de comportamentos complexos, como leitura e nomeação (Greer $\&$ Ross, 2008).

Sundberg e Partington (1998) sugerem que o foco principal de um programa de intervenção para crianças com autismo ou déficits de comunicação deveria ser no desenvolvimento efetivo de habilidades sociais e de linguagem, pois muitos comportamentos inadequados diminuem quando a criança adquire alguma linguagem. Adicionalmente, esses autores também sugerem que $o$ ensino de um operante pode facilitar a emergência de outros, como quando a criança emite uma resposta sob controle da pergunta "O que é isso?" (intraverbal) e de um objeto (tato). Na presente pesquisa, por exemplo, ao usar a dica ecoica no ensino dos outros operantes verbais, é possível que o repertório de imitação vocal tenha sido fortalecido, o que pode facilitar a aquisição de novas topografias de respostas vocais.

\section{CONSIDERAÇÕES FINAIS}

Entende-se que o objetivo desse estudo foi alcançado, o instrumento escolhido para avaliações e planejamento de ensino (VB-MAPP) demonstrou-se adequado e a intervenção comportamental de 8 horas semanais produziu importantes ganhos comportamentais para os três participantes.

O ensino de múltiplos operantes simultaneamente permitiu um desenvolvimento global dos participantes, principalmente nas áreas de linguagem (por exemplo, mandos, tatos e intraverbais) e de habilidades sociais (por exemplo, brincar, seguir instruções e imitar outras pessoas).

A principal limitação dessa pesquisa refere-se ao número de horas semanais de intervenção, principalmente, quando o participante apresenta pouco repertório verbal, essencialmente a fala. Estudos futuros podem ter uma duração maior, principalmente, quando o participante apresenta déficits mais severos, como a não existência da fala. Adicionalmente, estudos que utilizem delineamentos experimentais que exerçam maior controle sobre as variáveis (com grupo controle ou delineamentos de linha de base múltipla) podem atribuir maior valor aos resultados encontrados.

\section{REFERÊNCIAS}

American Psychiatric Association [APA]. (2013). Diagnostic and statistical manual of mental disorders (DSM-V). Washington: American Psychiatric Association.

Bloh, C. (2008). Assessing transfer of stimulus control across learners with autism. The Analysis of Verbal Behavior, 24(1), 87-101.
DeLeon, I. G.; Iwata, B. A. (1996). Evaluation of a multiplestimulus presentation format for assessing reinforcer preferences. Journal of Applied Behavior Analysis, 29(4), 519-533.

Gomes, C. G. S.; Souza, D. G.; Silveira, A. D.; Oliveira, I. M. (2017). Intervenção Comportamental Precoce e Intensiva com Crianças com Autismo por Meio da Capacitação de Cuidadores. Revista Brasileira de Educação Especial, 23(3) 377-390.

Greer, R. D.; Ross, D. E. (2008). Verbal behavior analysis: Inducing and expanding complex communication in children with severe language delays. Boston: Allyn \& Bacon.

Howard, J. S.; Stanislaw, H.; Green, G.; Sparkman, C. R.; Cohen, H. G. (2014). Comparison of behavior analytic and eclectic early interventions for young children with autism after three years. Research, 35(12), 3326-3344.

Lovaas, O. I. (1987). Behavioral treatment and normal educational and intellectual functioning in young autistic children. Journal of Consulting and Clinical Psychology, 55(1), 3-9.

Martone, M. C. C.; Santos-Carvalho, L. H. Z. (2012). Uma revisão dos artigos publicados no Journal of Applied Behavior Analysis (JABA) sobre comportamento verbal e autismo entre 2008 e 2012. Revista Perspectivas em Análise do Comportamento, 3(2), 73-86.

Schopler, E.; Reichler, R. J.; Bashford, A.; Lansing, M. D.; Marcus, L. M. (1990). Individualized assessment and treatment for autistic and developmentally disabled children. Vol. 1. Psychoeducational profile-revised (PEP-R). Austin, Tx: PRO-ED.

Skinner, B. F. (1957). Verbal Behavior. New York: Appleton Century - Crofts.

Smith, T. (2001). Discrete trial training in the treatment of autism. Focus on autism and other developmental disabilities, 16(2), 86-92.

Sundberg, M. L. (2008). Verbal behavior milestones assessment and placement program: The VB-MAPP. Concord, CA: AVB Press.

Sundberg, M. L.; Partington, J. W. (1998). Teaching language to children with autism and other developmental disabilities. Danville, CA: Behavior Analysts, Inc.

Szatmari, P.; Bryson, S. E.; Boyle, M. H.; Streiner, D. L.; Duku, E. (2003). Predictors of outcome among high functioning children with autism and Asperger syndrome. Journal of Child Psychology and Psychiatry, 44(4), 520-528.

Venter, A.; Lord, C.; Schopler, E. (1992). A follow-up study of high-functioning autistic children. Journal of Child Psychol Psychiatry, 33(3), 489-507.

Volkert, V. M.; Lerman, D. C.; Trosclair, N.; Addison, L.; Kodak, T. (2008). An exploratory analysis of task-interspersal procedures while teaching object labels to children with autism. Journal of Applied Behavior Analysis, 41(3), 335350.

Werner, E.; Dawson, G.; Munson, J.; Osterling, J. (2005). Variation 
in Early Developmental Course in Autism and its Relation with Behavioral Outcome at 3-4 Years of Age. Journal of Autism and Developmental Disorders, 35, 337-350.
Williams, L. C. A.; Aiello, A. L. R. (2001). O Inventário Portage Operacionalizado: Intervenção com famílias. São Paulo: Memnon.

Os autores agradecem e reconhecem o apoio constante da Coordenação de Aperfeiçoamento de Pessoal de Nível Superior - Brasil (CAPES) - CAPES/PROEX № do Processo: 23038.005155/2017-67. Esse trabalho é parte da dissertação de mestrado da primeira autora, defendida no Programa de Pós-Graduação em Educação Especial (PPGEEs) da Universidade Federal de São Carlos (UFSCar).

Recebido: 30 de outubro de 2018 Aprovado: 19 de dezembro de 2019 